\title{
Analytical Approach in Terms of Lead and Lag Parameter to Tune Database Performance
}

\author{
Bindu Sharma \\ M.tech Scholar \\ Department of computer \\ Science \\ A.I.T.M,Palwal
}

\author{
Mahesh Singh \\ Assistant Professor \\ Department of computer \\ Science \\ A.I.T.M,Palwal
}

\begin{abstract}
Performance tuning in database management system means escalating the performance of database by reducing time. For enhancing performance, analysis is important and analysis can be performed by neural network learning to save time spent in doing repeated work. Because neural network has ability to adapt dynamically varying environment .In this paper, working on two aspects is done and named as lead and lag parameters. Lead parameter is target and lag parameters are levers that need to press for achieving the target.

For identification of lead parameters; consider the criticality of the parameter (thru cardinality estimation) and lag parameters are the parameters that are associated with it and their time of processing affect lead parameter. This paper is all about analyzing the lag parameter and feeding only those lag parameters which are contributing in high share of time to automated tuning system.
\end{abstract}

\section{General Terms}

Lead parameter, Lag parameter, analysis.

\section{Keywords}

Performance tuning in database management system, Analysis of time parameters for tuning database performance.

\section{INTRODUCTION}

Database is an important part of any organization whether it is bank, airport, selling, university, factory, officialdom or telecommunications. Each and every industry relies on database. Database can be OLTP and OLAP.OLTP is very fast where performance is measured in terms of transaction per second but OLAP and BI are used for analysis where data takes time to give output and management for any analysis relies on BI or OLAP.

The agenda of this paper to bring a new concept to analyze the lag parameter and use only those parameters which are affecting the major portion of pie-chart drawn for time for that particular lag parameter. This will surely facilitate DBA at back end and management to access data in a very short span. The concept of BI is clear that we can have macro level view and we can drill down to any level to have micro level view. This needs to extract data from various disks and we will not go into this as this is part of SGA and DBA.

This paper is just presenting a new approach for analyzing the data and provides only that data which has high impact factor on time pie chart.

This is quite strategically wherein there is a need to identify only those parameters which are performers and what all are the parameters which are pushing them not to perform good. Lead parameters are those parameters which are the target and lag parameters are those parameters by focusing on them, can lead to target.

Lead and lag parameters are concept of management which can be applied in database for analysis purpose so that decision making become easier and effective.

Using lead and lag parameters in analysis and applying them correctly will lead to high response time.

Response time is defined as time taken by any query to give output after it got fired .By increasing the response time, it will be very easy to achieve customer centricity at very high scale and customer centricity is highly desirable to run any business effectively. The customer here is management and management can take effective decision based on the data extracted through BI or OLAP.

Neural network is used at e very great extent so that repetition can be overcome by smart approach that neural network has by adapting the dynamically varying environment.

This paper limits the discussion till analysis approach and repeating the same every time by using neural network learning and proving only those parameters to tune which has high impact factor on time pie chart for a given target/lead parameter.

The paper contains following sections. Section 2 present Literature Survey, section 3 presents proposed work, section 4 presents conclusion.

\section{LITERATURE SURVEY}

A far-reaching literature survey has been done to arrive at this approach and this literature survey has been very beneficial from all the aspects considered. This extensive literature survey belong to the below mentioned research papers.

These research papers were highly beneficial and we have taken many approaches into account and derived this idea. The literature review is presented as follows:

In the paper of Gaozheng Zhang, he has presented a model for application oriented database performance tuning and describes the main parameters affect the performance .This helped me in arriving at query level analysis [5].

In the paper of David J. Montana and Lawrence Davis on Training feedforward neural network using genetic algorithms, this paper illustrates the improvement gained by using genetic algorithm [2].

In the paper of S.F. Rodd and Dr. U.P.Kulkarni on "Adaptive tuning algorithm for performance tuning of database management system ",presents how the neural network adapt dynamically changing input and how we can use them for performance tuning [4]. 
In the paper of SreeKumar Vobugari, D.V.L.N. Somayajulu on "A model for building Dynamic indexes and storage and reuse of optimal query plans generated thru progressive optimization", they have presented the idea to reusing the concept of query plans by building dynamic indexes and this approach is extension of Robust query processing through progressive optimization. Cardinality estimation is also used in this [1].

In the paper of Hitesh Kumar Sharma and Aditya Shastri on "Architecture of Automated database tuning using SGA parameters" presents addressing the challenge of total cost of ownership is by making information system more self managing [3].

From the paper of Biplob K. Debnath on Statistical approach for ranking database tuning parameters, we have arrived to the conclude cardinality estimation for ranking and analyzing

\section{PROPOSED WORK}

In this paper the proposed work will be based on two parameters i.e. lead parameter and lag parameter.

Lead parameter are those parameters which needs to be considered, means whose output (time) is to be minimized i.e. to be considered for minimizing the time.

Lag parameters-what all are the levers that need to be pressed to achieve the lead parameter.

Lead parameter can be easily found out by criticality associated with it i.e. based on cardinality estimation.

Let's find out a parameter which has response time $\mathrm{T}$, and call it as lead parameter.

For a given query, we need to minimize the time of this query i.e. Told $>\mathrm{T}$ new, this is lead parameter means target.

To achieve this target, need to tune the following parameters:

$\mathrm{u}, \mathrm{v}, \mathrm{w}, \mathrm{x}, \mathrm{y}, \mathrm{z}$

These are lag parameters

Tuning all these parameters will lead to more time consumption.

It is better to calculate the percentage of time consumption (i.e. share of time each parameter WRT total time taken). And tune only those parameters which are contributing in $70 \%$ of the total time. FMS analysis of all the parameters in terms of time is required.

F-70\% of the time consumed to execute these parameters M- $20 \%$ of the time consumed to execute these parameters S- $10 \%$ of the time consumed to execute these parameters This paper will focus to tune only F category to increase the performance in terms of time.

Table 1: represents dynamic categorization of FMS analysis of the parameters

\begin{tabular}{|l|l|l|l|l|}
\hline $\begin{array}{l}\text { Parameters } \\
\text { used in any } \\
\text { query }\end{array}$ & $\begin{array}{l}\text { Arrange } \\
\text { in } \\
\text { descendin } \\
\text { g order }\end{array}$ & $\begin{array}{l}\text { Share } \\
\text { in } \\
\% \text { age }\end{array}$ & $\begin{array}{l}\text { Calculate } \\
\text { cumulative } \\
\text { share }\end{array}$ & $\begin{array}{l}\text { FMS } \\
\text { categorizat } \\
\text { ion }\end{array}$ \\
\hline $\mathrm{x}$ & 0.50 & $50 \%$ & $50 \%$ & $\mathrm{~F}$ \\
\hline $\mathrm{y}$ & 0.15 & $15 \%$ & $65 \%$ & $\mathrm{~F}$ \\
\hline $\mathrm{z}$ & 0.15 & $15 \%$ & $80 \%$ & $\mathrm{M}$ \\
\hline $\mathrm{w}$ & 0.10 & $10 \%$ & $90 \%$ & $\mathrm{M}$ \\
\hline $\mathrm{u}$ & 0.05 & $5 \%$ & $95 \%$ & $\mathrm{~S}$ \\
\hline $\mathrm{v}$ & 0.05 & $5 \%$ & $100 \%$ & $\mathrm{~S}$ \\
\hline Total & 1.00 & & & \\
\hline
\end{tabular}

\section{Flow of work:}

Target (Told $>$ Tnew)

\{

IITo achieve this, tuning of the following is required

If $(\mathrm{u}=\mathrm{F})$

Then tune (u) $\$ tuning can be done through self automated tuners or can be forwarded as input for performance tuning Else not_tune (u)

If $(\mathrm{v}=\mathrm{F})$

Then tune $(\mathrm{v})$

Else not_tune (v)

If $(\mathrm{w}=\mathrm{F})$

Then tune (w)

Else not_tune (w)

If $(x=F)$

Then tune $(\mathrm{x})$

Else not_tune(x)

If $(\mathrm{y}=\mathrm{F})$

Then tune(y)

Else not_tune(y)

If $(\mathrm{z}=\mathrm{F})$

Then tune $(\mathrm{z})$

Else not_tune (z)

\}

II the whole process is carried by neural network because neural network has ability to adapt dynamically changing inputs.

\section{The work can be summarized as:}

Identify lead parameter

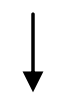

Check all the lag parameters associated with lead parameter and arrange in descending order of time share

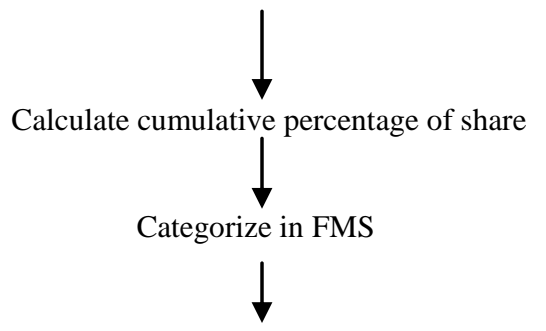

Tune only F category

\section{CONCLUSION}

Within our own perspective, we always have a feeling that we are working at the best possible level. However, it is important to do a reality check. Analysis is very important and by analyzing your work you will get to know the insights and can improve upon the same. And if you do analysis but you do not use it for betterment then again it is of no worth. The work proposes a frame work how to analyze the performance to two important parameters i.e. lead and lag parameters

The inspiration of this paper is based on extensive literature review. The ideas are from many papers are combined with a new approach and bit different analytical approach

This work can be extended for further analysis and for limited scope and can directly be implemented. It is just a part of tuning approach 


\section{REFERENCES}

[1] Sreekumar Vobugari, D.V.L.N. Somayajulu, and B.M. Subraya ,2012, A model for building dynamic indexes \& storage and Re-use of optimal query plans Generated thru progressive Optimization

[2] David J. Montana and Lawrence Davis, Training Feedforward Neural Networks Using Genetic Algorithms.

[3] Hitesh Kumar Sharma, Aditya Shastri , Ranjit Biswas, 2012, Architecture of Automated Database Tuning Using SGA parameter.

[4] S.F. Rodd, Dr, U.P. Kulkarni , 2010, Adaptive Tuning Algorithm for performance Tuning of database Management System

[5] Gaozheng Zhang, Mengdong Chen, Lianzhong Liu, A model for Application -oriented Database performance Tuning

[6] Debnath, B. K.; Lilja, D. J.; Mokbel, M. F., SARD: A Statistical Approach for Ranking database Tuning parameters, Data Engineering Workshop, 2008. ICDEW 2008. IEEE 24th International Conference, April 2008.
[7] Sanjay Agarwal, Nicolas Bruno, Surajit Chaudhari, AutoAdmin: Self Tuning Database System Technology, IEEE Data Engineering Bulletin, 2006.

[8] Chaudhuri, S.; Weikum G, Foundations of Automated Database Tuning, Data Engineering, April 2006.

[9] Michael L. Rupley, 2008. Jr. Introduction to Query Processing and Optimization. Indiana University at South Bend..

[10] Surjit Choudhuri, Vivek Narasayya, Self Tuning Database Systems: A Decade progress, Microsoft Research. 2007.

[11] Gerhar Weikum, Axel Moenkerngerg et. al. , Self-tuning Database Technology and Information Services :From wishful thing to viable Engineering, Parallel and Distributed Information System 1993.

[12] Gennadi Rabinovitch, David Wiese, Non-linear Optimization of Performance functions Autonomic Database Performance Tuning, IEEE Conference, 2007.

[13] Satish, S. K. ; Saraswatipura, M. K. ; Shastry, S. C, DB2 Performance Enhancements using Materialized Query Table for LUW Systems, 2007. ICONS '07. Second International Conference, April 2007. 